\title{
Comprehension of prosody in Huntington's disease
}

\author{
Lynn J Speedie, Nancy Brake, Susan E Folstein, Dawn Bowers, Kenneth M Heilman
}

\begin{abstract}
Patients with Huntington's Disease (HD) who were without dementia were compared to unilateral stroke patients and controls as previously reported in 1983, to discover if they had a prosodic defect. Subjects were presented tape-recorded speech filtered sentences and asked to indicate the tone of voice as happy, sad or angry (affective prosody), or as a question, command or statement (propositional prosody). HD patients were impaired in comprehension of both types of prosody compared to controls but were not different from stroke patients. A second study compared early HD patients with at-risk siblings and spouse controls on comprehension of affective and propositional prosody, discrimination of both types of prosody, rhythm discrimination and tonal memory (Seashore tests). HD patients were impaired in both comprehension and discrimination of all types of prosody. HD patients were less accurate than at-risk patients on the tonal memory task but not on the rhythm discrimination task. These findings suggest compromise in ability to understand the more subtle prosodic aspects of communication which may contribute to social impairment of HD patients very early in the course of the disease.
\end{abstract}

Department of Psychiatry and Behavioural Sciences, Johns Hopkins

University, Baltimore School of Medicine, Maryland.

LJ Speedie

N Brake

SE Folstein

Department of

Neurology, College of Medicine, University

of Florida, and the

Veterans

Adminstrative

Medical Center,

Gainsville, Florida,

United States.

D Bowers

KM Heilman

Correspondence to: $\mathrm{Dr}$ Speedie, Department of Geriatrics, Division of Neriatrics, Division of Neurogeriatrics, Ezrath Jerusalem, Israel.

Jerusalem, Israel. in revised form 9 October

1989.

Accepted 3 November 1989.
Huntington's disease (HD) may be associated with both cortical (frontal and temporal) and basal ganglia degeneration, especially the caudate. While the basal ganglia were considered to mediate primarily motor functions, it is now recognised that the basal ganglia are composed of multiple systems or circuits, some of which may not be primarily motor. DeLong and Georgopoulos ${ }^{1}$ for example, proposed two loops, a putaminal that was primarily motor and a caudate, that was mainly "complex" or associative.

Patients with HD experience more than motor symptoms. In addition to memory disorders they may also commonly have emotional symptoms. ${ }^{2}$ Emotions have both experiential and cognitive aspects. One of the cognitive aspects of emotions is the ability to comprehend emotional prosody. Prosody refers to the variations in stress and pitch that convey shades of meaning separate from the actual words said. ${ }^{3}$ The purpose of this study was to learn if patients with HD are impaired in the ability to comprehend prosody.

\section{Study one}

We compared HD patients who were in the early stage of their illness with both the stroke patients reported by Heilman et $\mathrm{al}^{4}$ and non brain-damaged controls for ability to comprehend affective and linguistic prosody.

Subjects The eight right hemisphere damaged (RHSD), nine left hemisphere damaged (LHD) and 15 patients without neurological disease (NHD) were all male and right handed. These patients were described by Heilman et al. ${ }^{4}$ Inclusion in the hemisphere damaged group was restricted to patients with cerebral infarction confined to one hemisphere as defined by computer tomography.

The six HD patients (five male) were all right handed, diagnosed within the past three years, and with a Mini Mental Status Exam (MMSE) score of 24 or more (above the demented range). They were employed without a decrease in their previous responsibilities or were still the primary homemaker. There was no past or present evidence of psychiatric illness according to DSM III criteria.

Stimuli The stimulus tape used in this study was described by Heilman et al. ${ }^{4}$ The speaker's voice was male. The stimulus tape for the propositional prosody condition consisted of 30 sentences of three to six seconds in duration (10 each of interrogative, imperative and declarative) presented in random order. The tape was filtered with a graphic spectrum equaliser (Model 124, B K Instruments) deleting frequencies $<100 \mathrm{~Hz}$ and $>5000 \mathrm{~Hz}$ and attenuating intervening frequencies as follows: $\quad 100 \mathrm{~Hz}=+5, \quad 125 \mathrm{~Hz}=0, \quad 160$ $\mathrm{Hz}=-5, \quad 200 \mathrm{~Hz}=10, \quad 250 \mathrm{~Hz}=0$, $315 \mathrm{~Hz}=-5,5000 \mathrm{~Hz}=-15$. The words were therefore unintelligible while the prosody remained.

The stimulus tape for the affective prosody condition consisted of 10 items each of happy, sad and angry intoned sentences and was also speech filtered so that the words could not be recognised. These sentences were presented in random order. The length of items was also three to six seconds in duration.

Procedure Testing was performed with patients seated in a quiet room. Half the subjects received the emotional prosody task first and the other half received the propositional prosody task first. Patients were informed that they would be unable to understand the words spoken because of the speech filtering process and that they should listen for the speaker's tone of voice. For the emotional prosody condition, the patients were told that the speaker's tone of voice 
would be either happy, sad or angry and that they were to indicate which tone of voice they thought the speaker was using by pointing to a response card. On the response card there were vertically arranged line drawings of a man's face appearing happy, sad or angry with the appropriate labels printed below each face. For the propositional prosody condition, the patients were told that the tone would be either a question, command or statement and that they were to indicate their choice by pointing to a response card on which were the symbols "?", "!" and "." with the appropriate labelling words. Verbalised responses were also accepted.

\section{Results}

For the emotional prosody task, the dependent variable was the number of affectively intoned sentences correctly identified. A oneway between groups analysis of variance (ANOVA) was performed using group (HD, RHD, LHD, NHD) as the between subjects factor. Results of this ANOVA reached a significant effect for group $(F=8.773$, $\mathrm{p}=0.000$ ). Post hoc comparisons using $t$ tests revealed that the HD patients were significantly less accurate $(\overline{\mathrm{X}}=65.7 \%)$ than controls $(\overline{\mathrm{X}}=87.3 \%, \mathrm{t}=2.754, \mathrm{p}=0.002)$ in identifying emotional prosody. However, the HD patients did not significantly differ from either RHD $(\overline{\mathrm{X}}=52.5 \%)$ or LHD $(\bar{X}=78.9 \%$ ) patients in their ability to comprehend affective prosody.

For the propositional prosody tasks, the dependent variable was the number of correctly identified sentences. Results of a between groups ANOVA again revealed a significant main effect for group $(F=11 \cdot 101$, $\mathrm{p}=0.000$ ). Post-hoc comparisons (t-tests) again indicated that the $\mathrm{HD}$ patients were significantly less accurate $(\overline{\mathrm{X}}=65 \%)$ than controls $(\bar{X}=89.7 \%, t=3.422, p=0.003)$, but were not different from LHD $(\overline{\mathbf{X}}=$ $59.8 \%)$ or RHD ( $\overline{\mathrm{X}}=60.3 \%)$ patients in their ability to comprehend propositional prosody.

\section{Discussion}

HD patients showed decreased comprehension of emotional prosody relative to nonneurologically ill patients. They also showed decreased ability to comprehend propositional prosody relative to the control patients. They were not different from the two unilateral stroke groups in their ability to comprehend emotional or propositional prosody. Thus, it would appear that very early in the course of HD, patients who are only mildly compromised and still professionally active appear to have difficulty comprehending both propositional and affective prosody to a degree similar to that seen in patients who have had unilateral cerebral infarctions.

Patients with unilateral right hemisphere lesions have expressive and/or comprehesion deficits for affective prosody. ${ }^{4-6}$ More specifically, there appears to be a prosodic perceptual defect. ${ }^{7}$ Perhaps a more basic per- ceptual processing disability is also contributing to the poor performance of HD subjects in identifying prosody. We therefore wanted to perform a prosody discrimination task where HD subjects did not have to "denote" the prosody but had to be able to tell if two filtered prosodic sentences sounded the same or different.

Prosody, even in filtered speech, is composed of several elements including the rhythm or cadence of variations as well as changes in tone or melody of the speech sounds. We also wanted to learn if $\mathrm{HD}$ patients could perform tasks requiring accurate rhythm or tonal discrimination. Finally, we also wanted to discover if atrisk relatives of HD patients have difficulty comprehending prosody.

\section{Study two}

This study investigated (a) whether HD patients could discriminate prosody; (b) whether patients at risk for $\mathrm{HD}$ also have difficulty comprehending prosody; and (c) whether these deficits might be related to elements of prosody such as rhythm or tone.

Subjects Six HD patients who met the criteria for inclusion in study one were recruited. Seven at-risk siblings and seven spouse controls were also recruited. While all subjects were above the MMSE score range for dementing illnesses, the HD patients were lower than either of the other groups (table 1). One HD patient from the first study participated in study two, with an intervening interval of seven months.

Stimuli Six tasks were administered to these subjects. Task 1 tested comprehension of affective prosody. A five minute excerpt was taken from the affective comprehension stimuli described in study one. It contined 21 speech filtered sentences, seven each of happy, sad and angry tone which were arranged in random order. Task 2 tested comprehension of propositional prosody and was a similarly constructed five minute excerpt of propositional prosodic items from study one.

Task 3 tested discrimination of affective prosody. Using the stimuli from task 1 , a five minute discrimination task was constructed in which pairs of filtered sentences were presented. The task consisted of 12 items in which each tone (happy, sad, or angry) was paired with a different sentence of happy, sad or angry tone. Of the twelve, six were of the same tone and six were different and the order of these was randomised. Task 4 tested discrimination of propositional prosody and was similarly constructed based on the sentences used in task 2 .

Task 5 was the Rhythms Test and the sixth

Table 1 Demographic and MMSE characteristics

\begin{tabular}{llllll}
\hline & $H D$ & $A R$ & Spouse & $F$ & $P$ \\
\hline Age & 45.8 & 35.4 & 38.3 & 1.201 & 0.33 \\
Education & 13.3 & 14.9 & 14.4 & 0.412 & 0.67 \\
MMSE & 26.3 & 29.8 & 29.2 & 7.437 & 0.005 \\
\hline
\end{tabular}


Table 2 Mean per cent correct responses of early HD, at-risk and spouses

\begin{tabular}{|c|c|c|c|c|c|}
\hline & $H D$ & $A R$ & Spouse & $F$ & $P$ \\
\hline $\begin{array}{l}\text { Affective comprehension } \\
\text { Propositional comprehension } \\
\text { Affective discrimination } \\
\text { Proportional discrimination } \\
\text { Rhythm discrimination } \\
\text { Tonal memory }\end{array}$ & $\begin{array}{l}54 \cdot 0 \\
64 \cdot 0 \\
77 \cdot 0 \\
59 \cdot 0 \\
74 \cdot 0 \\
44 \cdot 0\end{array}$ & $\begin{array}{l}71 \cdot 0 \\
86 \cdot 0 \\
80 \cdot 0 \\
84 \cdot 0 \\
83 \cdot 0 \\
77 \cdot 0\end{array}$ & $\begin{array}{l}79 \cdot 0 \\
86 \cdot 0 \\
92 \cdot 0 \\
84 \cdot 0 \\
79 \cdot 0 \\
60 \cdot 0\end{array}$ & $\begin{array}{l}3 \cdot 701 \\
3 \cdot 480 \\
3 \cdot 175 \\
6 \cdot 601 \\
1 \cdot 136 \\
3 \cdot 867\end{array}$ & $\begin{array}{l}0.045 \\
0.053 \\
0.066 \\
0.008 \\
0.345 \\
0.040\end{array}$ \\
\hline
\end{tabular}

task was the Tonal Memory task from the Seashore Measures of Musical Talents. ${ }^{8}$

Procedures The order of task presentation was varied so that half of the subjects received a prosody task first while the other half received a Seashore task first. Initial presentation of a propositional or affective prosody task was also varied. The task instruction for the prosody identification tasks was as described in study one. For the discrimination task, subjects were told to decide if the speaker was using the same tone of voice (for example, both angry statements or both questions) or whether the voice tone was different (a happy and a sad item or a question and a command) and to indicate their response by saying "same" or "different" or by pointing to the response card on which these words were printed. The Seashore tasks were administered according to their respective directions. The rhythm task requires subjects to listen to a pair of 5,6 or 7 beat rhythmic patterns and to indicate if the rhythms are the same or different. The tonal memory task requires the subject to indicate which of 4,5 or 6 notes in each pair is different.

\section{Results}

For each of the six tasks a one way between groups analysis of variance (ANOVA) was performed. The dependent variable was the number of correct responses to the task and the between subjects factor was group (HD, at-risk, spouse control). Post hoc comparisons utilised $t$ tests (least squares difference).

The groups were different in ability for comprehending emotional prosody (table 2). Post hoc comparisons indicated that HD patients performed worse than the spouse controls $(t=2.672, p=0.021)$. Results of the ANOVA for the propositional prosody comprehension suggested that HD patients performed worse than either the at risk patients $(t=2.341, p=0.035)$ or spouse controls $(t=2 \cdot 314, p=0.039)$.

Differences in ability to discriminate affective prosody approached significance with the HD patients performing worse than spouses $(t=2.352, p=0.037)$. HD patients also had much greater difficulty discriminating propositional prosody than either at-risk subjects $(t=3 \cdot 187, p=0.009)$ or spouse controls $(\mathrm{t}=3 \cdot 187, \mathrm{p}=0.009)$.

Accuracy of performance on the rhythm discrimination task was not significantly different for any of the groups. However, HD patients did have more difficulty than at-risk patients on the tonal memory task $(t=2 \cdot 384$, $\mathrm{p}=0.035)$.

\section{Discussion}

In addition to the same comprehension deficits seen in study one, the HD patients in the second study also showed an inability to discriminate either type of prosody. While the HD subjects included in the study were early in the course of their disease and had as yet suffered no significant functional decline, their MMSE scores were lower than those of the at risk and control subjects. Thus their failures on tasks of prosodic comprehension and discrimination may simply reflect that these tasks are more sensitive measures of subtle but global cognitive deterioration.

However, they were able to discriminate rhythmic patterns reasonably well. Since the duration of the rhythm items as well as the total length of the task are equivalent to the other tasks, it seems unlikely that problems of attention and concentration or general dementia per se could explain their failure on prosodic tasks.

There is some suggestion that tonal aspects of prosody may be problematic for HD patients. However, since the tonal memory task does have a greater memory component, the question of whether the difficulty of the task or tonal perception is the basis for the deficit remains unclear.

Even though still functional and professionally active, HD patients were impaired on prosodic tasks, with the severity of impairment approaching that seen in stroke patients. These findings suggest that there may be cognitive or perceptual changes which compromise ability to understand the more subtle aspects of communication and which contribute to social impairment early in the course of the disease.

Milner ${ }^{9}$ compared patients with right and left temporal lobectomies on the tonal and rhythm sections of the Seashore Musical Abilities Test. Following either left or right temporal lobectomy, patients showed little change on the rhythm subtest compared to their preoperative ability. However, following right but not left temporal lobectomy, patients had much more difficulty on the tonal memory subtest. Heilman et $a l,{ }^{410}$ and Tucker et $a l^{7}$ showed that right hemisphere damage impairs comprehension and discrimination of emotional prosody. These in addition to other studies of emotional prosody and tonal patterns in normal subjects suggest a special role of the right hemisphere in tonal patterns and in prosodic comprehension (especially emotional). Our findings in this study together with the previous study suggest that perhaps early in their disease, patients with HD may resemble patients with right hemisphere dysfunction.

Structural changes in HD appear to involve primarily caudate and putamen. Positron emission tomography using the fluorine-18labelled fluorodeoxyglucose method revealed decreased glucose metabolism in the caudate nucleus and in the putamen of patients with very early HD. Metabolism in cortical and other subcortical regions was normal. ${ }^{11}$ The caudate, however, receives projections from 
virtually all major cortical areas and in addition afferent and efferent projections link the caudate and the substantia nigra. ${ }^{12}$

Recently, Scott et al ${ }^{13}$ found that patients with Parkinson's disease with substantia nigra dysfunction also have difficulty interpreting the prosodic aspects of speech. The fact that both diseases show deficits in prosodic comprehension may suggest a role for these subcortical structures.

We thank Dr Russell $M$ Bauer for making the tapes and Dr Howard Rothman for speech-filtering these tapes.

1 DeLong MR, Georgopoulos AP. Motor functions of the basal ganglia. In: Brookhart JM, Mountcastle VB, Brooks VB, eds. Handbook of physiology, Sect 1, The nervous system, Vol 2, Motor control, Pt 2. Bethesda: Am Physiol Soc, 1981:1017-61.

2 Mayneux R. Emotional changes associated with basal ganglia disorders. In: Heilman KM, Satz P, eds. Neuropsychology of human emotion. New York: Guilford Press, 1983:141-64.
3 Monrad-Krohn G. The prosodic quality of speech and its disorders. Acta Psychol Scand 1947;22:225-65.

4 Heilman KM, Bowers D, Speedie L, Coslett HB. Comprehension of affective and nonaffective prosody. Neurology 1984;34:917-21.

5 Ross $E$. The aprosodias: functional-anatomic organization of the affective components of language in the right hemithe affective components of language

6 Weintraub S, Mesalum M, Kramer L. Disturbance in prosody: a right hemisphere contribution to language. Archs Neurol 1891;38:742-4.

7 Tucker D, Watson R, Heilman K. Affective discrimination and evocation in patients with right parietal disease. Neurology 1977;27:947-50.

8 Seashore CE, Lewis D, Saetveit DL. Seashore measures of musical talents (Rev ed). New York: Psychological Corporation, 1960.

9 Milner B. Laterality effects in audition. In: Montcastle VB, ed. Interhemispheric relations and cerebral dominance. Baltimore: Johns Hopkins University Press, 1962:177-95.

10 Heilman K, Scholes R, Watson R. Auditory affective agnosia: disturbed comprehension of affective speech agnosia: disturbed comprehension of affective

11 Kuhl DE, Phelps ME, Markham CJ, Mettler EJ, Riege WJ, Winter J. Cerebral metabolism and atrophy in Huntington's disease determined by 18 FDG and computed tomographic scan. Ann Neurol 1982;12:425-34.

12 Brodal A. Neurological anatomy in relation to clinical medicine. New York: Oxford University Press, 1981.

13 Scott S, Caird F, Williams B. Evidence for an apparent sensory speech disorder in Parkinson's disease. J Neurol Neurosurg Psychiatry 1984;47:840-3. 